\title{
Ben Johnstons Verhältnis zu Harry Partch und seine Three Chinese Lyrics
}

\section{Ben Johnston's Relationship with Harry Partch and his Three Chinese Lyrics}

Johnston's compositional journey began with a momentous imposition: when he was twelve, he was dragged to a lecture on Hermann von Helmholtz's influence on Claude Debussy. The speaker demonstrated the pure intervals and the phenomenon of partials on a monochord. For Johnston, however, his most significant artistic encounter was with Harry Partch, whose apprentice he was for six months in 1950. After that, it took almost ten years before he started composing in just intonation.

In retrospect, the amalgamation of European traditions with just intonation and the respective redefinition of tonality can be considered Johnston's main achievement. He took the first important steps towards this compositional approach in Three Chinese Lyrics. In most of his works of the 1950s, Johnston had used a neoclassical idiom, which remains here only in rudimentary form. Instead, he uses free atonal techniques, which already presage key pieces of his in the 1960s. He does not yet use micro-intervals here, and he offers no explicit instructions for using just intonation. But given the instruments he chose, many passages can only be imagined in pure intonation. This article shows that this can be seen as anticipating Johnston's characteristic use of just intonation. Whereas Partch was concerned with the development of a new sound system, Johnston sought to expand the existing one.

\section{Ben Johnston und die Just Intonation}

Es begann mit einer folgenreichen Zumutung: Als Zwölfjähriger wurde Ben Johnston (1926-2019) zu einem Vortrag über den Einfluss von Hermann von Helmholtz auf Claude Debussy mitgeschleppt. Der Referent demonstrierte an einem Monochord die reinen Intervalle und das Phänomen der Teiltöne. Johnston war tief beeindruckt: »I never lost the feeling of mystery and unfolding new possibilities that world of simple mathematical ratios opened to me. « $^{1}$ Anlässlich eines Porträtkonzertes veröffentlichte der Richmond Times Dispatch am 30. Januar 1944 ein aufschlussreiches Porträt des knapp 18-Jährigen. Eigens betont wurde,

DOI: $10.26045 /$ po-020

1 Ben Johnston: Beyond Harry Partch [1981], in: "Maximum Clarity« and other Writings on Music, hg. von Bob Gilmore, Urbana/Chicago: University of Illinois Press, 2006, S. 243-250, hier S. 245. 
dass dieser dem wissenschaftlichen Zugang zur Komposition verpflichtet sei und an eine bevorstehende große Revolution der Musik glaube, an der er sich zu beteiligen gedenke: "with the clarification of the scale which physics has given to music there will be new instruments with new tones and overtones. >And I shall write for them.... People will not like this new music - not at first. It will be so different - but it will be more nearly perfect. « ${ }^{2}$ Dies muss als Absichtserklärung verstanden werden, denn es wird noch 16 Jahre dauern, bis Johnston mit Just Intonation zu komponieren beginnt, und das ganze Frühwerk wirkt wenig revolutionär. ${ }^{3}$ Erstaunlicherweise konnte er zu dieser Zeit mit den Ideen von Harry Partch noch nicht vertraut sein, denn dessen Buch Genesis of a Music wurde erst 1949 publiziert. ${ }^{4}$ Als Johnston auf die soeben erschienene Schrift stieß, fiel diese auf entsprechend fruchtbaren Boden. Im Anschluss an die Lektüre schrieb er dem Autor einen enthusiastischen Brief und erkundigte sich, ob es möglich wäre, bei ihm Komposition zu studieren. ${ }^{5}$ Partch antwortete, dass er es nicht möge, als Lehrer bezeichnet zu werden, er würde ihn aber als Lehrling annehmen. Zudem warnte er ihn ausdrücklich, mit einer Frau anzureisen, denn laut seinem Biografen Bob Gilmore hätte er ihn gerne auch als seinen Liebhaber gesehen: ${ }^{6}$ Partch »warned that Johnston should not become biologically trapped. $\ll^{7}$ Johnston indessen ignorierte den Befehl. Nach Abschluss seines Masters in Musiktheorie am Cincinnati Conservatory reiste er im August 1950 mit seiner Frau Betty nach Gualala (Kalifornien). Er war sich an das akademische Umfeld gewohnt und begierig, mit Partch dessen Theorien zu diskutieren. Diesem hingegen lag dies nicht und er hätte Fragen oft mit dem Kommentar »Haven't you read the book? ${ }^{8}{ }^{8}$ abgeschmettert. Trotzdem wurde diese Begegnung für Johnston zur folgenreichsten seiner künstlerischen Laufbahn. Das Verhältnis indes gestaltete sich schwierig. Partch war ruppiger als erwartet, unverhohlen unfreundlich, und er fuhr fort, nackt im Garten zu arbeiten und im ganzen Haus »ungeheuerliche phallische Symbole « ${ }^{9}$ zu verteilen. Zudem reagierte er, der

Heidi Von Gunden: The Music of Ben Johnston, Metuchen, NJ/London: The Scarecrow Press, 1986, S. 5 f.

3 Die erste Komposition Johnstons in Just Intonation sind die Five Fragments from Thoreau's Walden (1960/61).

4 Harry Partch: Genesis of a Music. Monophony. The Relation of Its Music to History, Madison: University of Wisconsin Press, 1949. In der Folge zitiert wird die erweiterte zweite Auflage: ders.: Genesis of a Music. An Account of a Creative Work, Its Roots and Its Fulfillments. Second Edition, Enlarged, New York: Da Capo Press, 1974.

5 Bob Gilmore: Harry Partch. A Biography, New Haven/London: Yale University Press, 1998, S. 188.

6 Ebd., S. 193.

$7 \quad$ Von Gunden: The Music of Ben Johnston, S. 11.

8 Vgl. ebd., S. 12.

$9 \quad »[$...] egregiously phallic symbolism in my house«. Vgl. Brief von Harry Partch an Larry Marshall vom 27. August 1949, zit. nach Philip Blackburn: Enclosure 3. Harry Partch, Saint Paul, MN: American Composers Forum, 1997, S. 119. 
starke hypochondrische Züge aufwies, extrem aufgebracht, wenn das Essen nicht pünktlich serviert wurde, was ihm die - auf die Minute geplante - Einnahme seiner diversen Medikamente erschwerte..$^{10}$ Die Johnstons sahen sich in Gualala mit einer Menge harter, körperlicher Arbeit konfrontiert. Zuallererst hatten sie eine alte Hirtenhütte herzurichten, die ihnen fortan als Behausung dienen würde. Dieses primitive Leben war für beide eine neue Erfahrung, und Ben hatte nach eigenem Bekunden zwei linke Hände: »I am - or was then - the sort of person who could not pick up a hammer without dropping it on my foot. $"{ }^{11}$ Aber der ungeschickte Lehrling verfügte über ein feines Gehör, konnte die Intervalle akkurat reproduzieren und verstand Partchs Theorien beinahe ohne Erklärungen. Partch »began to grasp that there might be some sort of real convergence between us. ${ }^{12}$

$\mathrm{Zu}$ Johnstons Aufgaben gehörte es, die Partch-Instrumente täglich zu stimmen. Zudem lernte er, sie zu spielen, nahm an den regelmäßig stattfindenden Proben teil und wirkte zusammen mit Betty bei der Aufnahme zweier Stücke mit, den Eleven Intrusions und Dark Brother. ${ }^{13}$

Während seiner Zeit in Gualala erhielt Johnston von Wilford Leach eine Anfrage, die Bühnenmusik zu einem neuen Stück zu schreiben. Er hatte bereits früher mit Leach zusammengearbeitet und überzeugte Partch, die Musik gemeinsam zu komponieren. Entstanden ist aus dieser Zusammenarbeit das Tonbandstück The Wooden Bird, »some twenty minutes' worth of incidental music, most of it short fragments of songs, music for scene changes, and sound effects. «14

Nach wenigen Monaten endete der Aufenthalt Johnstons in Gualala abrupt. Betty und er mussten überstürzt abreisen, weil Partch allergisch auf einen Zeckenbiss reagiert hatte und sich in Fort Bragg behandeln lassen musste.

Auf Anraten Partchs begann Johnston im Anschluss an seinen Aufenthalt in Gualala ein Kompositionsstudium bei Darius Milhaud am Mills College in Oakland. Um dies möglich zu machen, hatte Partch im Rahmen einer Dinnerparty von Agnes Albert ein vorgängiges Treffen mit Milhaud arrangiert. ${ }^{15}$ Direkt nach dem Studium erhielt Johnston im Alter von 26 Jahren einen Lehrauftrag an der University of Illinois in Urbana-Champaign und blieb dieser Institution bis zu seiner Emeritierung treu. Obschon er sich in den vollgepackten ersten Jahren seiner Dozentur kompositorisch nicht mit der Just Intonation auseinandersetzte, blieb der Kontakt zu Partch

11 Ben Johnston: The Corporealism of Harry Partch [1975], in: »Maximum Clarity« and other Writings on Music, hg. von Bob Gilmore, Urbana/Chicago: University of Illinois Press, 2006, S. 219-231, hier S. 228.

12 Ebd., S. 229.

13 Diese Aufnahmen sind auf diversen späteren Schallplatten zu finden. Vgl. Thomas McGeary: The Music of Harry Partch. A Descriptive Catalog, New York: Institute for Studies in American Music, Conservatory of Music, Brooklyn College of the City University of New York, 1991, S. $164-182$.

14 Gilmore: Harry Partch. A Biography, S. 195.

15 Vgl. Von Gunden: The Music of Ben Johnston, S. 13. 
bestehen. Er setzte sich regelmäßig dafür ein, Aufführungen von dessen Musik zu ermöglichen, auch wenn dieser ihn als Komponisten abgeschrieben hatte: »But since it took me about ten years to do anything concrete with what I had learned from him, he had long since given up on me. ${ }^{16} \mathrm{Ab} 1955$ bemühte sich Johnston um eine Aufführung von The Bewitched, einer Tanz-Satire von Partch. Die beiden ersten angefragten Choreografinnen sagten ab, offenbar weil Partch als kompliziert im Umgang galt. ${ }^{17}$ Die Wahl fiel schließlich auf Alwin Nikolais, einen der führenden Choreografen seiner Zeit. Johnston hatte sich wohl erhofft, Partch damit einer breiteren Öffentlichkeit bekannt zu machen, aber die Zusammenarbeit erwies sich als schwierig: »I once committed a serious artistic gaffe. I got Partch to work with Alwin Nikolais. There could not have been a worse combination of collaborators. Partch and Nikolais were poles apart. « ${ }^{18}$ Es war Johnstons undankbare Aufgabe, zwischen den beiden zu vermitteln und die Produktion zusammenzuhalten, »with both men struggling at times, and with Partch fighting to prevent its taking place. ${ }^{19} \mathrm{Um}$ die Aufführung erst möglich zu machen, hatte er eigens einen Kredit aufgenommen, in der Hoffnung, diesen mit den Ticketeinnahmen wieder tilgen zu können. Auch dies hielt Partch nicht davon ab, aus Unzufriedenheit mit der choreografischen Umsetzung mit einer Absage der Uraufführung zu drohen. Immerhin kam die Aufführung zustande und Johnston konnte später eine zweite Produktion an der Washington University in St. Louis bewirken.

Trotz der nervenaufreibenden Zusammenarbeit engagierte er sich weiterhin für Partch und konnte im Verlauf der nächsten Jahre zwei Kompositionsaufträge für ihn erwirken. Revelation in the Courthouse Park und Water! Water! wurden beide an der University of Illinois uraufgeführt. ${ }^{20}$

Während eines Sabbaticals begann sich Johnston 1959 in Theorie und Praxis wieder mit der Just Intonation zu beschäftigen und konzentrierte sich in der Folge auf sein eigenes Komponieren. Die Bemühung zur Verbreitung des Werks von Partch intensivierte er erst nach dessen Tod 1974 wieder. »As a first step, Partch's work can be and must be brought before a wider public and his significance correctly assessed, not diminished to the level of a cultural oddity. $\aleph^{21}$ Begünstigt wurde dieses neuerliche Engagement durch zahlreiche Einladungen anderer Universitäten. Nebst den organisatorischen Bemühungen und dem Appell an den Kulturbetrieb, hat Johnston Partch auch künstlerisch gewürdigt. Im String Quartet No. 4, Ascent, Amazing Grace (1973) zitiert er ausführlich aus der First Greek Study und das 1975

19 Ebd.

20 Revelation in the Courthouse Park wurde im Rahmen des Festival of Contemporary Arts am 11. April 1961 uraufgeführt; Water! Water! am 9. März 1962. 
komponierte In Memory für Streicher, acht Schlagzeuger, Tonband und Dias entstand im Gedenken an die Tänzerin Margaret Erlanger und Partch.

Bevor Johnstons langer Kampf gegen die Parkinsonerkrankung begann, kehrte er zum Schluss seines Komponierens ganz zu Partch zurück. Unter seinen letzten Werken finden sich zwei Arrangements für das Kronos-Quartett: Partchs Barstow und U. S. Highball für Sprecher und Streichquartett. Laut Heidi Von Gunden übernahm Johnston bei einigen Konzerten die Rolle des Sprechers und ließ sich, um sein Äußeres demjenigen von Partch anzugleichen, eigens einen Bart wachsen. ${ }^{22}$

Das Verhältnis Johnstons zu Partch lässt sich als beständiges Ringen bezeichnen, als ein Wechselspiel zwischen Bewunderung und der Suche nach einer eigenen ästhetischen Sprache. Er machte es sich dabei zur Aufgabe, die Impulse von Partch weiterzuentwickeln: "As an even more important second step, those of us who can must carry on aspects of his work in directions of which, perhaps, he never dreamed or felt himself ill-equipped to deal with. ${ }^{23}$ Anders als Partch gab Johnston nicht vor, sich von den europäischen Musiktraditionen zu distanzieren, sondern war bestrebt, diese mit der Just Intonation zu verbinden. Johnston dachte nicht in Stammtonhöhen, sondern entwickelte ein modulares Tonhöhensystem mit stetig wechselnden Intervallkombinationen. Dabei ließ er das von Partch gesteckte $>11$ Limit $<$ hinter sich und benutzte auch höhere Teiltöne als konstituierende Intervalle. ${ }^{24}$ Zudem war er nicht an der Entwicklung neuer Instrumente interessiert, sondern suchte nach Wegen, die Just Intonation mit einem herkömmlichen Instrumentarium zu realisieren.

\section{Three Chinese Lyrics für Sopran und zwei Violinen (1955)}

Partch selber hatte Johnston prophezeit, er würde nicht zum Jünger werden: »He said I was too much like him and I would have to find my own way. ${ }^{25}$ Als Johnston 1955 seine drei chinesischen Lieder komponierte, befand er sich noch mitten in dieser Suche. In den ersten Berufsjahren schrieb er mehrere Bühnenstücke, eine Kammeroper zu Gertrude Stein und zwei Stücke für Jazzband. Insgesamt entstanden in den 1950er Jahren gegen dreißig Werke, wobei sich Johnston bei den allermeisten eines neoklassizistischen Idioms bediente. Erst während eines Sabbaticals (1959/60), wandte er sich wieder der Just Intonation zu. Rückblickend wirken die Three Chinese Lyrics wie eine Vorwegnahme der in den 1960er Jahren zentralen kompositorischen Aspekte. ${ }^{26}$ Mit Ausnahme weniger neoklassizistischer Rudimente verlässt er

22 Persönliches Interview mit Heidi Von Gunden, geführt von Marc Kilchenmann, 5. September 2019.

23 Johnston: Beyond Harry Partch, S. 249.

24 Mit `Limit`bezeichnet Partch die mit dem höchsten verwendeten Teilton gebildeten Intervallverhältnisse.

25 Johnston: Beyond Harry Partch, S. 250.

26 Vgl. Bob Gilmore: Ben Johnston. A Chronology, in:»Maximum Clarity«and other Writingson Music, hg. von Bob Gilmore, Urbana/Chicago: University of Illinois Press, 2006, S. xxv-xxviii, hier S. xxix. 
sich nicht länger auf die Sicherheit des vertrauten Idioms "and began to experiment with pitch and timbre. $\ll^{27}$ Insbesondere finden sich zahlreiche Passagen in freier Atonalität, ein Verweis auf Werke der 1960er Jahre, in denen er die Verbindung von Reihentechniken und Just Intonation anstrebt.

Die Gedichte von Li Bai kannte Johnston spätestens seit seiner Zeit in Gualala, denn seine Ehefrau Betty setzte dort Li Bais Gedicht The Jewel Stairs' Grievance für Stimme und Gitarre. ${ }^{28}$ Mit demselben Text beginnt Johnston seine Trilogie:

\section{The Jewel Stairs' Grievance (Rihaku [Li Bai], übers. Pound)}

The jewelled steps are already quite white with dew,

It is so late that the dew soaks my gauze stockings,

And I let down the crystal curtain

And watch the moon through the clear autumn..$^{29}$

Das nur 18 Takte lange Stück ist durchgehend in einem langsamen 7/16-Takt gehalten. Obschon die Binnen-Unterteilung ständig wechselt, wirkt das Stück metrisch ungebunden und erhält einen stets fließenden, schwebenden Charakter. Johnston unterstreicht damit die kontemplative Erzählhaltung. Dazu passend wird durch unterschiedlich lange Formteile und eine ungleiche Takteinteilung der Violinen und des Gesangs das Entstehen eines periodischen Zeitgefühls erschwert. Zwar beginnt jede Textzeile mit einem Auftakt von 3/16, mit Ausnahme der ersten Zeile fällt die betonte Silbe aber nicht mit der Eins des Taktes zusammen.

Die Violinen eröffnen das Stück mit einer dissonanten Kontrapunktik, die durchgehende Sechzehntel ergibt. Die beiden Linien bewegen sich hauptsächlich in Sekund- und Terzbewegungen, wobei sich die erste Violine durch den gelegentlichen Einschub von Septimen bis zum a\#"' hinaufschraubt. Nach dem Vorspiel setzt der Sopran mit einer schlichten Linie ein, ebenfalls in beinahe durchgehenden Sechzehnteln, mit spärlichen Haltenoten und einer einzigen Unterteilung (Abbildung 1).

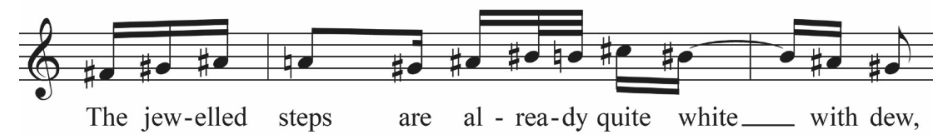

Abb. 1: The Jewel Stairs' Grievance, T. 5-7 $7^{30}$

Von Gunden: The Music of Ben Johnston, S. 26.

Vgl. ebd., S. 27.

Ezra Pound: Cathay, London: Mathews, 1915, S. 13.

Die Abbildungen wurden vom Autor erstellt. Sie folgen hauptsächlich einer sehr schlechten Kopie im Privatbesitz von Heidi Von Gunden. Das Stück wurde zwar durch Independent Music Publishers, N. Y. verlegt, der Verlag ist aber nicht mehr existent. Die einzige auffindbare Kopie dieser Ausgabe liegt in der Duke University Library in Durham, NC. Die im August 2019 offiziell über die SNB bestellte Kopie ist zum Zeitpunkt der Drucklegung noch nicht eingetroffen. Im Nach- 
Diese formale Anordnung wird nach der ersten Textzeile wiederholt, nur dass der Sopran nun melodisch ausladend auf das Zwischenspiel der beiden Violinen antwortet (Abbildung 2).

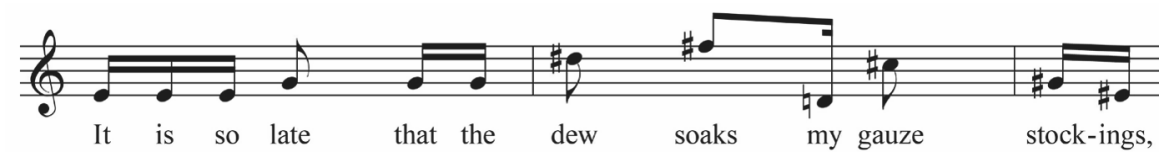

Abb. 2: The Jewel Stairs' Grievance, T. 9-11

Eine ähnliche Behandlung der Stimme finden wir auch beim nächsten Zeilenpaar, doch fasst Johnston dieses zusammen und verzichtet auf ein Zwischenspiel. Indem er nun die Stimme solistisch einsetzt, wird die unterschiedliche Melodieführung noch deutlicher.

Formal lässt sich das Stück als A - B - A' - B' beschreiben. Getrennt werden die beiden Zeilenpaare durch einen Einschub der zweiten Violine, im halben Tempo. Von Gunden vergleicht das Stück mit einer chinesischen Schriftrolle, deren spärliche, ja suggestive Textur den Zuhörenden einlädt, die Bedeutung selber zu liefern. ${ }^{31}$ Das Stück endet mit einem Dur-Quartsextakkord in extrem weiter Lage $\left(g-c^{\prime}-e^{\prime \prime \prime}\right)$, der dadurch nur bedingt tonal klingt.

Taking Leave of a Friend (Rihaku [Li Bai], übers. Pound)

Blue mountains to the north of the walls,

White river winding about them;

Here we must make separation

And go out through a thousand miles of dead grass.

Mind like a floating wide cloud,

Sunset like the parting of old acquaintances

Who bow over their clasped hands at a distance.

Our horses neigh to each other as we are departing. ${ }^{32}$

Die erste Violine beginnt das Stück auf derselben Tonhöhe, mit der sie das erste Lied

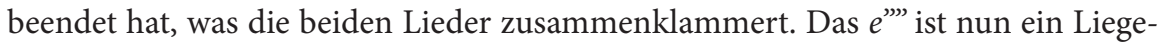
ton, der zum entrückten Grundton einer einfachen, zwischen E-Dur und e-Moll changierenden Melodie der 2. Violine wird (Abbildung 3).

lass von Ben Johnston an der Northwestern University Music Library findet sich zudem ein Fragment einer anderen Kopie. Auf diese wurde so weit als möglich zurückgegriffen.

31 Vgl. Von Gunden: The Music of Ben Johnston, S. 27.

32 Pound: Cathay, S. 28 f. 


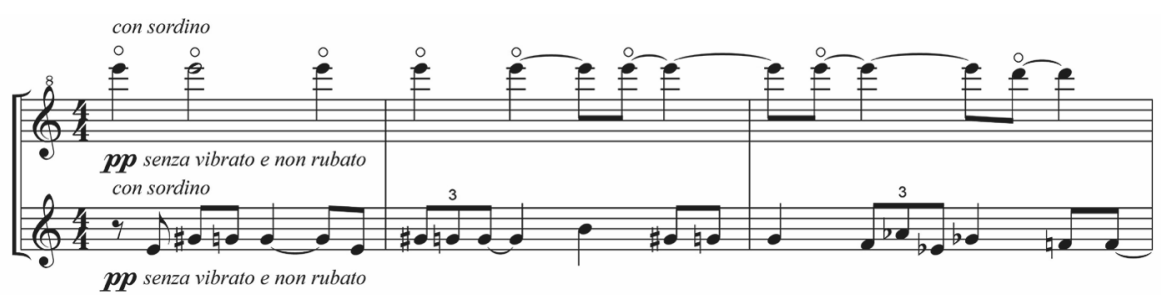

Abb. 3: Taking Leave of a Friend, T. 1-3

Johnston reichert im weiteren Verlauf die Melodie mit Sekunden an, den einfachen Duktus eines Trauermarsches behält er in den Violinen aber während des ganzen Stücks bei - IIn Stillness and Space $«{ }^{33}$ als würden sich die beiden Freunde schon voneinander entfernen. Durch die Stimmführung in extrem weiter Lage wird diese Trennung dann auch akustisch erfahrbar gemacht.

Nimmt das Vorspiel mehr als einen Viertel der Gesamtdauer ein, folgen danach vier Textzeilen in beinahe lapidarer Beiläufigkeit. Über weite Strecken ist die Stimme unbegleitet komponiert, was wiederum das Gefühl der Separation unterstreicht. Wie im ersten Lied changiert Johnston erneut zwischen einer am Sprechgesang orientierten Schreibweise und einer expressiven Stimmbehandlung (Abbildung 4).

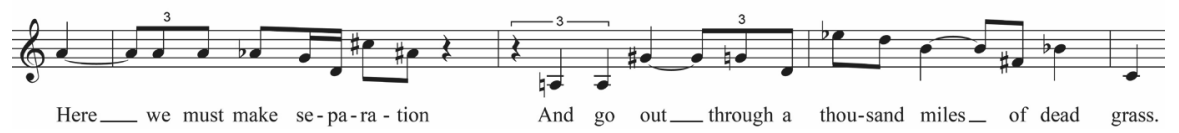

Abb. 4: Taking Leave of a Friend, T. 10-14

Durch diese von den Violinen unterschiedene Deklamationshaltung wird eine zusätzliche innere Spannung erzeugt. Sind die ersten drei Zeilen unbegleitet, wird die vierte durch Liegetöne in höchster Lage eingefärbt $\left(d^{\prime \prime \prime}+e^{\prime \prime \prime}\right)$, ein Effekt, den Von Gunden mit weißem Rauschen assoziiert. ${ }^{34}$ Auf diesen Höhepunkt folgt ein kurzes Zwischenspiel mit durchwegs fallenden kleinen Sekunden in der ersten Violine. Diese eindringliche Seufzer-Motivik wird dadurch unterstützt, dass die Mehrzahl der Vorhalte auf kleine Terzen aufgelöst wird, was die Hoffnungslosigkeit der beiden Freunde bei der Trennung zu betonen scheint (Abbildung 5). ${ }^{35}$

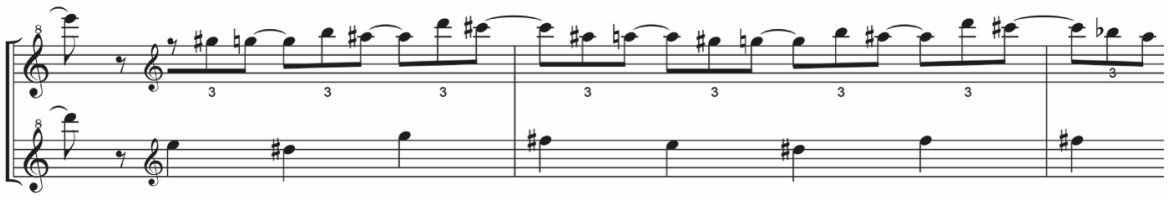

Abb. 5: Taking Leave of a Friend, T. 14-16

33 Tempobezeichnung des 2. Liedes.

34 Vgl. Von Gunden: The Music of Ben Johnston, S. 28.

35 Ebd. 
Die fünfte Textzeile setzt in diese Seufzer-Motivik ein, während die sechste wiederum unbegleitet ist. In einem zweiten kurzen Zwischenspiel nimmt Johnston das Anfangsmotiv wörtlich wieder auf. Die abschließende Textzeile wird analog zur vierten mit einer großen Sekunde in der viergestrichenen Oktave begleitet.

Lament of the Frontier Guard (Rihaku [Li Bai], übers. Pound)

By the North Gate, the wind blows full of sand,

Lonely from the beginning of time until now!

Trees fall, the grass goes yellow with autumn.

I climb the towers and towers to watch out the barbarous land:

Desolate castle, the sky, the wide desert.

There is no wall left to this village.

Bones white with a thousand frosts,

High heaps, covered with trees and grass;

Who brought this to pass?

Who has brought the flaming imperial anger?

Who has brought the army with drums and with kettle-drums?

Barbarous kings.

A gracious spring, turned to blood-ravenous autumn,

A turmoil of wars-men, spread over the middle kingdom,

Three hundred and sixty thousand,

And sorrow, sorrow like rain.

Sorrow to go, and sorrow, sorrow returning,

Desolate, desolate fields,

And no children of warfare upon them,

No longer the men for offense and defence.

Ah, how shall you know the dreary sorrow at the North Gate,

With Rih[a]ku's name forgotten,

And we guardsmen fed to the tigers. ${ }^{36}$

Die beiden ersten Lieder wurden stilistisch ähnlich behandelt und wirken durch die Klammer des $e^{\text {"’” }}$ wie ein Paar. Das dritte Lied setzt sich davon deutlich ab. Das Vorspiel ist ein wilder Tanz im 6/8-Takt mit durchgehenden gezupften Achteln. Die beiden Violinen spielen dabei einen Hoquetus, wobei einzig die Taktanfänge zusammenfallen (Abbildung 6). 


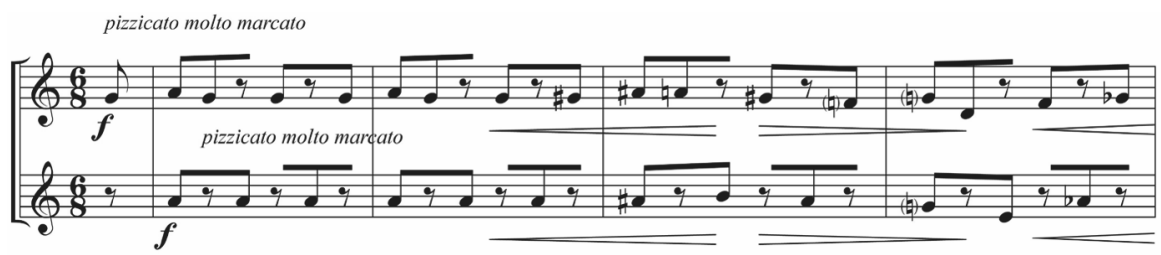

Abb. 6: Lament of the Frontier Guard, T. 1-4

Hauptsächlich in Sekundschritten kreist Johnston 11 Takte um das a', bevor er die

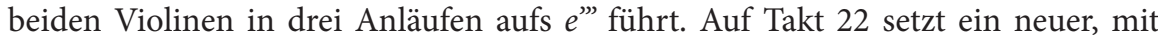
"rhapsodic« bezeichneter Formteil ein, der mit seinem heiter-trotzigen Charakter an osteuropäische Tanzmusik erinnert. Auf einen raumgreifenden rhythmischen Impuls folgt über mehrere Takte eine fallende Linie im Diminuendo (Abbildung 7).

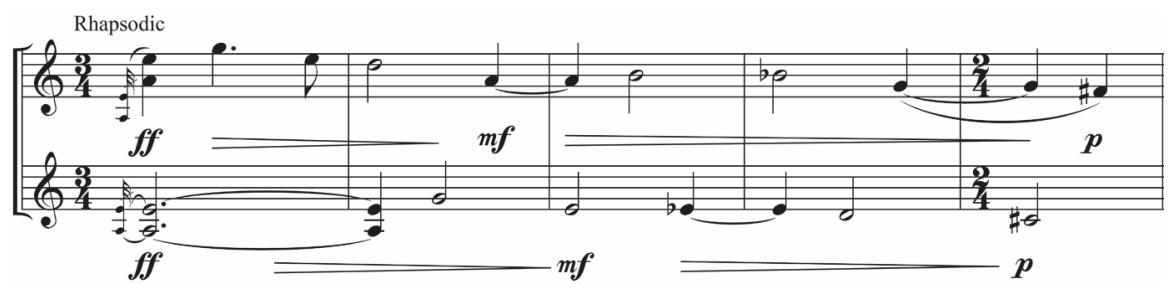

Abb. 7: Lament of the Frontier Guard, T. 22-26

Dieser Vorgang wiederholt sich dreimal mit geringfügigen Variationen. Auf die erste Repetition setzt im Takt 28 die Stimme ein, hochexpressiv, mit Oktavsprüngen im Forte. Das musikalische Geschehen steht damit in einem seltsamen Widerspruch zur Tristesse des Textes. Statt die Kontemplation des ersten und die Wehmut des zweiten Liedes weiter zu steigern, scheint Johnston hier die Wut des an die Tiger verfütterten Wachmanns zu komponieren.

Der ganze Beginn steht stellvertretend für die von Johnston in den 1950er Jahren benutzte neoklassizistische Sprache. In starkem Kontrast hierzu folgt ab Takt 43 ein chromatisches Kontinuum der ersten Geige, begleitet von gezupften Quarten und Quinten in der zweiten. Das Geschehen beruhigt sich wiederum nur scheinbar in einem Unisono auf d' (Abbildung 8).

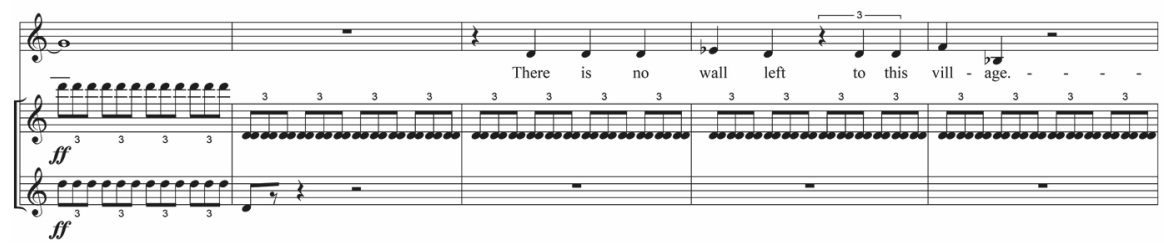

Abb. 8: Lament of the Frontier Guard, T. 54-58 
Die durchgehenden Achteltriolen vermitteln eine große innere Unruhe, die sich nach diesem energetischen Tiefpunkt erneut entlädt, hin zum zweiten Auftreten der Rhapsodie in Takt 74. Auf das letzte Erscheinen des Themenkopfes setzt in der ersten Violine ein hoher Triller ein, der acht Takte anhält und zur endgültigen Beruhigung führt. Auf »And sorrow, sorrow like rain « beginnt der Sopran solistisch den resignierten Schlussteil. Erst hier passt die Stimmung zum Text. Auf die drittletzte Zeile setzen die Violinen mit einem neuerlichen Trauermarsch in durchgehenden Vierteln ein. Beide Instrumente spielen dabei stets Doppelgriffe in Terzen, Quarten und Quinten. Der durchgehend vierstimmige Satz unterscheidet sich mit seinen Sekundreibungen deutlich vom Trauermarsch im zweiten Lied (Abbildung 9).

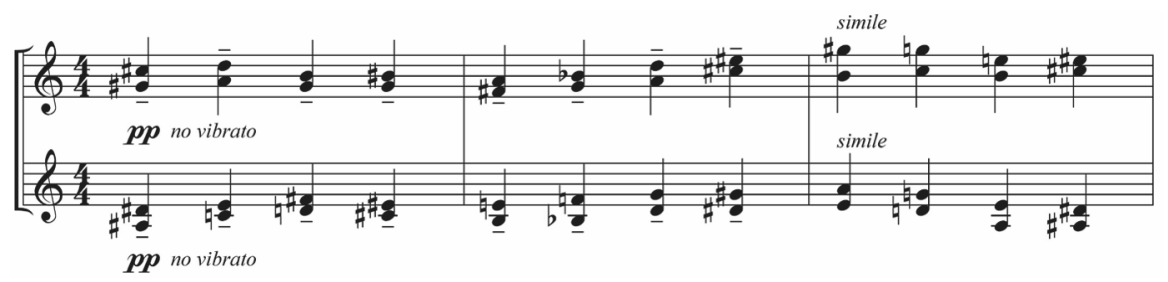

Abb. 9: Lament of the Frontier Guard, T. 106-108

\section{Eine Abgrenzung zu den Seventeen Lyrics by Li-Po von Harry Partch}

Die Seventeen Lyrics by Li Po sind die ältesten der von Partch aufbewahrten Kompositionen. ${ }^{37}$ In einer Art »adolescent auto-da-fé « ${ }^{38}$ hatte er alle älteren Stücke verbrannt. ${ }^{39}$ Gilmore zeigt auf, dass Partch einige der später zentralen Aspekte seines Komponierens zum Zeitpunkt der Niederschrift der Seventeen Lyrics noch nicht formuliert hatte. Dazu gehören Konzepte wie `Otonality` versus >Utonality` oder der geometrisch aufgebaute Tondiamant. ${ }^{40}$ Auch wenn in den Lyrics by Li Po Theorie und Praxis nicht deckungsgleich sind, ${ }^{41}$ sind die zentralen stilistischen Aspekte seines späteren Werkes hier schon anzutreffen. So folgt die Rhythmik dem natürlichen Sprachrhythmus und metrisch gebundene Rhythmen sind nur in Passagen ohne Gesang anzutreffen: ${ }^{42} » I$ came to the realization that the spoken word was the distinctive expression my constitutional makeup was best fitted for, and that I needed

Vgl. Bob Gilmore: On Harry Partch's Seventeen Lyrics by Li-Po, in: Perspectives of New Music 30/2 (1992), S. 22-58, hier S. 22.

38 Partch: Genesis of a Music, S. x.

39 Das Lied My Heart Keeps Beating Time war früher publiziert worden und überlebte die Zerstörung. Vgl. Pirate Paul [Pseudonym für Harry Partch]: My Heart Keeps Beating Time, San Francisco: Lloyd Campbell Publications, 1929.

40 Vgl. Gilmore: On Harry Partch's Seventeen Lyrics by Li-Po, S. 29.

41 Vgl. ebd., S. 25.

42 Vgl. ebd., S. 24. 
other scales and other instruments. $\aleph^{43}$ Dementsprechend bewegen sich die Lyrics by Li Po in einem äußerst engen Tonhöhenrahmen. Dies widerspiegelt Partchs Verachtung gegenüber dem Belcanto-Singen und dessen »devitalized tricks of >serious` singing ${ }^{44}{ }^{44}$ wie dem allgegenwärtigen gerollten R, Portamenti und der affektierten Stilisierung eines ıverfeinerten Englisch. ${ }^{45}$ Diese Attitüden wären allenfalls für europäische Zungen passend, den fürs amerikanische Englisch charakteristischen "gliding tones ${ }^{46}$ aber geradezu entgegengesetzt. Partch betrachtete seine Vertonungen als archaische Musik: »The ... lyrics by Li Po are set to music in the manner of the most ancient of cultured musical forms. $\aleph^{47}$ Dazu gehört die Vermeidung expressiver Gestik und wortwörtlicher Wiederholungen. ${ }^{48}$

Mit den Three Chinese Lyrics macht Johnston einen gewichtigen Schritt weg von Partch. Anders als dieser verwendet er nicht die eigene Stimme, sondern einen der Liedtradition der Zweiten Wiener Schule verpflichteten Sopran. Auch bei der Wahl der Begleitung setzt er sich deutlich ab und wählt zwei >traditionelle< Violinen anstelle der `neuen` Adapted Viola. Durch die Verwendung zweier Begleitinstrumente eröffnen sich kontrapunktische Möglichkeiten, die Partch durchwegs vermeidet. Dementsprechend verwendet er Elemente der klassischen Formenlehre, während Partch in den Augen Johnstons allen Arten von Avantgardismus misstraute und europäische Traditionen zurückwies. ${ }^{49}$ Johnston aber ist von allem Anfang an bestrebt, diese zu nutzen und mit den Errungenschaften Partchs zu verbinden:

Rather soon I felt that my eventual task would be to alter attitudes, especially theoretic currents within the mainstream, from the mainstream, to the mainstream. It would be my role to bring his work into relation with accepted traditions and recognized challenges to tradition, and to whatever extent necessary and possible to bring these enormous trends into relation to some of his most important achievements. ${ }^{50}$

Erste Spuren dieses Bestrebens finden sich in den Chinese Lyrics.

Nicht nur die kompositorischen Mittel unterscheiden sich, Johnston wählte auch eine andere Übersetzung. Während Partch die Version von Shigeyoshi Obata verwendete, entschied sich Johnston für diejenige von Ezra Pound. In beiden Fällen finden sich allerdings keine Begründungen für die getroffene Wahl, insbesondere keine Hinweise darauf, ob jeweils ästhetische Gründe den Ausschlag gegeben haben.

Partch: Genesis of a Music, S. 5.

44 Ebd., S. 52.

45 Vgl. ebd.

46 Ebd.

47 Zit. nach Gilmore: On Harry Partch's Seventeen Lyrics by Li-Po, S. 34.

48 Vgl. ebd., S. 36.

49 Vgl. Johnston: The Corporealism of Harry Partch, S. $222 \mathrm{f}$.

50

Ebd., S. 228. 
Gesichert scheint nur, dass im Falle Partchs keine persönlichen Gründe vorliegen, denn er lernte Pound erst nach der Niederschrift der Lyrics by Li Po kennen..$^{51}$ Ausgeschlossen werden kann zudem, dass Partch Johnston die Übersetzung Pounds empfohlen hätte, denn er fand letzteren "ra most difficult man<. Pound's >difficult nature, however, lay less in his musical or poetic attitudes than in the fact that by 1935 he was rarely willing to discuss any subject at length except economics or politics, which Partch must have found hard to endure. $\aleph^{52}$

Vielleicht wollte Johnston sich deutlich absetzen und wählte deshalb eine andere Übersetzung; bedenkt man aber den politischen Hintergrund, ist seine Wahl zumindest erstaunlich. Pound war Anhänger Mussolinis und weigerte sich auch nach Kriegsende, sich vom Faschismus zu distanzieren. 1943 wurde er in den USA wegen Landesverrat angeklagt und 1945 nach Einmarsch der amerikanischen Truppen in Italien festgenommen. Da er von einem Gutachter für geisteskrank erklärt worden war, entging er der möglichen Todesstrafe, saß aber die nächsten 12 Jahre in einer staatlichen Nervenheilanstalt ein. Zur Entstehungszeit der Three Chinese Lyrics machte sich der frisch zum Nobelpreisträger gekürte Ernest Hemingway für die Freilassung von Pound stark, was den implizit politischen Charakter der Textwahl von Johnston nicht schmälert.

Wichtiger als die unterschiedliche Übersetzung scheinen mir aber technische Aspekte zu sein, insbesondere die divergierende Behandlung der Stimme. Die unbegleiteten Stellen sind zwar metrisch notiert, animieren in ihrer Schlichtheit aber, sich rhythmische Freiheiten zu nehmen. Zudem ist der Ambitus der Stimme in diesen Passagen meist sehr eingeschränkt. In der Verbindung dieser beiden Aspekte kommen diese Abschnitte dem mikrointervallischen Sprechgesang Partchs zumindest nahe. Andererseits finden sich mehrere Stellen im Duktus der Zweiten Wiener Schule, mit großen Intervallen und Melismen (Abbildung 10).

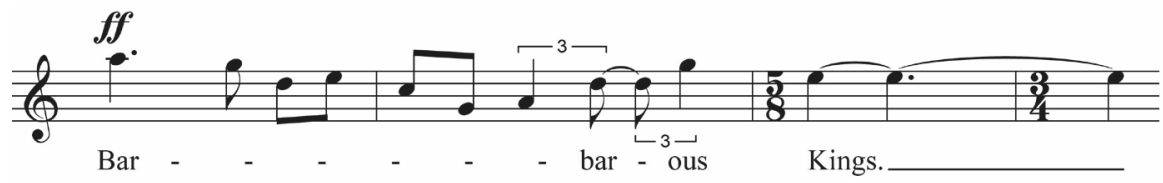

Abb. 10: Lament of the Frontier Guard, T. 73-76

Wie die Stimme werden auch die beiden Violinen unterschiedlich eingesetzt. Wechselt der Sopran zwischen expressivem Gesang und Sprechgesang, changieren die Geigen zwischen freier Atonalität und traditioneller Harmonik. Dass es Johnston gelingt, die Lieder trotz dieser Inhomogenität wie aus einem Guss klingen zu lassen, weist diesen die erwähnte Sonderstellung in seinem Frühwerk zu.

51 Vgl. auch den Beitrag von Eva Schestag in diesem Band, S. 191-203.

52 Gilmore: Harry Partch. A Biography, S. 109. 
Anders als Partch verwendet er keine Mikrointervalle und es finden sich keine expliziten Hinweise zum Einsatz der Just Intonation. Viele Stellen sind aber beim gewählten Instrumentarium nur rein intoniert vorstellbar. Dazu gehören der Beginn des zweiten Liedes (Abbildung 3), der Themenkopf der Rhapsodie (Abbildung 7), die hohen natürlichen Flageoletts oder die zahlreichen Doppelgriffe in Quarten respektive Quinten. Auch dies ist eine Vorwegnahme der spezifischen Verwendung der Just Intonation bei Johnston: Ging es Partch um die Entwicklung eines neuen Tonsystems, strebte Johnston die evolutive Erweiterung des bestehenden an. Die Amalgamierung europäischer Traditionen und der Just Intonation und damit verbunden die Neudefinierung der Tonalität sind das zentrale Verdienst Ben Johnstons. Die ersten Schritte hin zu diesem großen Ziel tat er in den Three Chinese Lyrics.

\section{Literatur}

Blackburn, Philip (Hg.): Enclosure 3. Harry Partch, Saint Paul, MN: American Composers Forum, 1997.

Gilmore, Bob: On Harry Partch's Seventeen Lyrics by Li-Po, in: Perspectives of New Music 30/2 (1992), S. 22-58, https://doi.org/10.2307/3090619.

Gilmore, Bob: Harry Partch. A Biography, New Haven/London: Yale University Press, 1998.

Gilmore, Bob: Ben Johnston. A Chronology, in: "Maximum Clarity« and other Writings on Music, hg. von Bob Gilmore, Urbana/Chicago: University of Illinois Press, 2006, S. xxvxxviii.

Johnston, Ben: The Corporealism of Harry Partch [1975], in: »Maximum Clarity« and other Writings on Music, hg. von Bob Gilmore, Urbana/Chicago: University of Illinois Press, 2006, S. 219-231.

Johnston, Ben: Beyond Harry Partch [1981], in: »Maximum Clarity « and other Writings on Music, hg. von Bob Gilmore, Urbana/Chicago: University of Illinois Press, 2006, S. $243-$ 250.

McGeary, Thomas: The Music of Harry Partch. A Descriptive Catalog, New York: Institute for Studies in American Music, Conservatory of Music, Brooklin College of the City University of New York, 1991.

Partch, Harry: Genesis of a Music. Monophony. The Relation of Its Music to History [First Edition], Madison: University of Wisconsin Press, 1949.

Partch, Harry: Genesis of a Music. An Account of a Creative Work, Its Roots and Its Fulfillments. Second Edition, Enlarged, New York: Da Capo Press, 1974.

Pirate Paul [Pseudonym für Harry Partch]: My Heart Keeps Beating Time, San Francisco: Lloyd Campbell Publications, 1929.

Pound, Ezra: Cathay, London: Mathews, 1915.

Von Gunden, Heidi: The Music of Ben Johnston, Metuchen, NJ/London: The Scarecrow Press, 1986. 
Marc Kilchenmann studierte Fagott, Komposition, Elementare Musikpädagogik und Research on the Arts. Ähnlich vielseitig wie seine Studien erweist sich sein Betätigungsfeld. In den letzten Jahren sind die Komposition und Improvisation verstärkt ins Zentrum gerückt. Verbunden wird dies mit musikwissenschaftlichen Forschungen im Bereich der Neuen Musik. Neben Studien zu Hermann Meier beschäftigte er sich mit der formalen Konstituierung des Gesamtwerkes von Jean Barraqué und schreibt zurzeit eine Dissertation zur Extended Just Intonation am Beispiel des US-amerikanischen Komponisten Ben Johnston. 


\title{
Der doppelte Po und die Musik
}

Rätoromanisch-chinesische Studien, besonders zu

Li Po, Harry Partch und Chasper Po

\author{
Herausgegeben von \\ Mathias Gredig, Marc Winter, \\ Rico Valär und Roman Brotbeck \\ Redaktionelle Mitarbeit \\ Daniel Allenbach
}

Königshausen \& Neumann 
Bibliografische Information der Deutschen Nationalbibliothek

Die Deutsche Nationalbibliothek verzeichnet diese Publikation in der Deutschen

Nationalbibliografie; detaillierte bibliografische Daten sind im Internet über http://dnb.d-nb.de abrufbar.

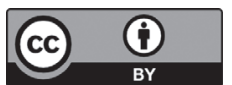

Dieses Werk ist lizenziert unter der Creative Commons Attribution 4.0 Lizenz (BY). Diese Lizenz erlaubt unter Voraussetzung der Namensnennung des Urhebers die Bearbeitung, Vervielfältigung und Verbreitung des Materials in jedem Format oder Medium für beliebige Zwecke, auch kommerziell. (Lizenztext: https://creativecommons.org/ licenses/by/4.0/deed.de) Die Bedingungen der Creative-Commons-Lizenz gelten nur für Originalmaterial. Die Wiederverwendung von Material aus anderen Quellen (gekennzeichnet mit Quellenangabe) wie z. B. Schaubilder, Abbildungen, Fotos und Textauszüge erfordert ggf. weitere Nutzungsgenehmigungen durch den jeweiligen Rechteinhaber.

Erschienen 2021 im Verlag Königshausen \& Neumann GmbH

(C) bei den Autoren

Die Druckvorstufe dieser Publikation wurde vom Schweizerischen Nationalfonds zur Förderung der wissenschaftlichen Forschung unterstützt.

\section{FNSNF}

SCHWEIZERISCHER NATIONALFONDS

ZUR FÖRDERUNG DER WISSENSCHAFTLICHEN FORSCHUNG

Wir danken der Kulturförderung des Kantons Graubünden.

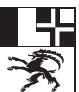

Kulturförderung Graubünden. Amt für Kultur

Promoziun da la cultura dal Grischun. Uffizi da cultura

Promozione della cultura dei Grigioni. Ufficio della cultura

SWIISSLOS

Hochschule der Künste Bern

www.hkb.bfh.ch

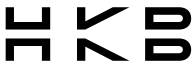

Hochschule der Künste Bern

Haute école des arts de Bern

Bern University of the Arts

Umschlag: skh-softics / coverart

Umschlagabbildung: Lea Gredig

Print-ISBN 978-3-8260-7180-5

PDF-ISBN 978-3-8260-7233-8

DOI $10.26045 /$ po

https.//doi.org/10.36202/9783826072338

Gedruckt auf säurefreiem, alterungsbeständigem Papier

Printed in Germany

www.koenigshausen-neumann.de

www.ebook.de

www.buchhandel.de

www.buchkatalog.de 


\section{Inhalt}

Prolog

Dumenic Andry

Chasper Pos Humor

Renzo Caduff

Chasper Pos rhythmische Versgestaltung - eine shinkende Mähre`?

Rico Valär

Rätoromanische Nachdichtungen chinesischer Lyrik bei

Gian Fadri Caderas und Peider Lansel

Eine Spurensuche

Mathias Gredig

China in rätoromanischen Zeitungen, Zeitschriften

und literarischen Texten

Marion Eggert

Schwalbenflug in Gedichten von Li Bai und Chasper Po

Thomas Geissmann

Die Rolle der Gibbons beim chinesischen Dichter Li Bai

Marc Winter

„Chinas Dichterfürst«

Die Rezeption Li Bais als literarischer Superstar im Westen

Eva Schestag

"A most difficult man«

Ezra Pound als Übersetzer von Li Bai, mit einem Seitenblick auf Shigeyoshi Obata

Odila Schröder

Chinesische Li-Bai-Vertonungen in Jahren der Unruhe 
Mathias Gredig

Quantitative Überlegungen zum Phänomen

der Li-Bai-Vertonungen im Westen

Mit Beobachtungen zu drei Vertonungen des Gedichtes

Chun ye Luo cheng wen di (In einer Frühlingsnacht in Luoyang eine Flöte hören)

\section{Gesine Schröder}

"Die Hüften schwingen sich nun nicht mehr»

Li-Bai-Vertonungen von Komponistinnen

Heinrich Aerni

Li-Bai-Vertonungen in der Schweiz

Matthias Schmidt

Übersetzung ohne Original?

Gustav Mahler, Anton Webern und Li Bai

\section{Christoph Haffter}

Szenen der Selbstenttäuschung

Hanns Eislers Die rote und die weiße Rose nach Li Bai und die Antinomien der Kriegslyrik

Thomas Meyer

„Wunderlich im Spiegelbilde»

Zu einigen Vertonungen des Pavillon-Gedichts

\section{Mathias Gredig}

Gedanken über Li Bais Jing ye si (Gedanken in einer stillen Nacht)

und dessen Vertonungen im Westen

\section{Martin Skamletz}

"I've turned into a great reviser."

Lee Hoibys Vertonung von Li Bais The River-Merchant's Wife: A Letter

und ihr Bezug zu Harry Partch

\section{Martin Skamletz}

"Of course I am a weak shadow of Lee Hoiby as a Kitharist."

Five letters by Harry Partch, 1948-1958

\section{Marc Kilchenmann}

Ben Johnstons Verhältnis zu Harry Partch und seine Three Chinese Lyrics 
Eleni Ralli

Parallelen und Modifikationen der Notation in verschiedenen Quellen

von Harry Partchs Seventeen Lyrics by Li Po

Schwierigkeiten und Transkriptionsvorschläge

Charles Corey

Gesture and Intention in the Art Songs of Harry Partch

Caspar Johannes Walter

Sprechmelodie als Quelle von Melodik und Harmonik

The Intruder aus Harry Partchs Li-Bai-Vertonungen

Roman Brotbeck

Der Sprechgesang bei Arnold Schönberg und Harry Partch

Eine Annäherung

Namensregister 